\title{
Chronocarto, un Web-SIG libre pour les archéologues (pas seulement...)
}

\section{Chronocarto, a web-GIS for archaeologists (but not only...)}

\author{
Dr. Michel Dabas ${ }^{1}$, Katherine Gruel ${ }^{2}$, Michel Cartereau ${ }^{3}$, Yadh Riahi ${ }^{4}$ \\ ${ }^{1}$ Chargé de Recherches au CNRS, UMR8546 -AOrOc (CNRS-ENS-EPHE), Université PSL, michel.dabas@ens.fr \\ ${ }^{2}$ Directrice de Recherche CNRS, PSL-ENS-AOrOc, UMR8546. katherine.gruel@ens.fr \\ ${ }^{3}$ Enseignant-chercheur AgroParisTech, michel.cartereau@agroparistech.fr \\ ${ }^{4}$ Directeur de Yebni Information et communication, contact@yebni.com
}

RÉSUMÉ. Chronocarto est un web-SIG développé depuis 2006 pour la représentation de données cartographiques en archéologie. II a été conçu pour permettre un travail de réflexion en réseau entre chercheurs, non spécialistes de SIG, depuis la phase d'acquisition jusqu'à celle de l'enrichissement de documents existants en ligne. La plateforme hébergée sur le TGIR Huma-Num supporte la notion de projet associée à une communauté d'utilisateurs dont les droits sont modulables par un administrateur. La notion de copyright a été introduite aussi bien au niveau de la donnée que pour les cartes. Chaque projet est constitué d'un certain nombre de cartes éventuellement regroupées dans des dossiers différents. Les données peuvent être spatiales (fichiers images géoréférencées, fichiers vecteurs mais aussi base de donnée ou simple tableur) ou non-spatiales (fichiers de traitement de texte, images, vidéos, liens Internet). L'archivage est journalier et incrémentiel. Le statut des projets peut évoluer: 'en projet', 'en cours', 'achevé', 'publiable en ligne (public)' et 'archivé' (non visible mais stocké pour une durée de 30 ans minimum). Actuellement, la plateforme gère plus d'une centaine de projets et plusieurs milliers de cartes. Certaines des cartes peuvent être associées à des Atlas thématiques grâce à des mots clefs. Les Atlas permettent d'interroger des bases de données grâce à des requêtes simples (Où, Quand? Quoi ?). Les résultats de l'interrogation sont sous forme de cartes de répartition. Chronocarto continue d'être développé en interne et son accès est libre.

ABSTRACT. Chronocarto is a web-GIS developed since 2006 for the representation of cartographic data in Archaeology. It has been designed to allow for networking among researchers, non-specialists in GIS, from the acquisition phase to the enrichment of existing on-line documents. The platform is hosted on the Huma-Num TGIR and supports the concept of projects associated with a community of users whose rights can be modulated by an administrator. The concept of copyright has been introduced both at the data level and for the maps. Each project consists of a number of maps possibly grouped in different folders. The data can be spatial (georeferenced image files, vector files but also database or simple spreadsheet) or non-spatial (word processing files, images, videos, Internet links). Archiving is daily and incremental. The status of projects may change: 'in project', 'in progress', 'completed', 'publishable online (public)' and 'archived' (not visible but stored for a minimum of 30 years). Currently, the platform manages over a hundred projects and several thousand maps. Some of the maps can be associated with thematic Atlas thanks to key words. The Atlas allows to query databases with simple questions like Where, When? What?. The results of the query are in the form of distribution maps. Chronocarto continues to be developed internally and its access is free.

MOTS-CLÉS. web-SIG, collaboratif, gestion de projet, copyright, atlas.

KEYWORDS. web-GIS, project management, collaborative, copyright, atlas.

Chronocarto désigne actuellement (2018) une plateforme de cartographie et d'analyse de données hébergées sur les serveurs de la TGIR (Très Grande Infrastructure de Recherche) Huma-Num ${ }^{1}$. Portée par le laboratoire AOrOc (UMR8546) depuis les années $2006^{2}$, il désignait au départ uniquement le web-SIG développé dans le cadre de l'ANR Celtecophys et transféré sous une licence ouverte, gage de pérennité, à l'ENS et ses partenaires. Cet article ne fait référence qu'à la partie web-SIG et ne

\footnotetext{
${ }^{1}$ https://www.huma-num.fr

2 Katherine Gruel et Michel Dabas, www.archeo.ens.fr

(c) 2019 ISTE OpenScience - Published by ISTE Ltd. London, UK - openscience.fr
} 
développe pas les autres outils de la plateforme (collections en ligne, moteur de recherche IOTA, extracteur d'informations textuelles, gazeteer).

Ce SIG open-source a été développé par la société Yebni (Tunis) comme une des retombées d'un programme de l'Agence Nationale de la Recherche (ANR Celtecophys) en 2006. Dans le cadre d'une approche spatiale des structures artisanales à l'âge du fer développé dans ce programme, les partenaires (AOrOc, Géocarta, INRAP, Université Paris VI- Sisyphe) avaient été amenés à étudier un certain nombre de sites archéologiques en France. Les données de prospection géophysique acquises sur le terrain et traitées sous forme de carte par Géocarta ont été versées sur le web-SIG Chronocarto (Dabas, 2016).

\section{Choix d'une technologie Web-SIG}

Le développement d'un web-SIG a été jugé nécessaire afin de répondre à deux attentes : comment gérer des données de plusieurs sites archéologiques simultanément et comment gérer des données lorsque beaucoup de partenaires interagissent sur le même projet? En effet la société Géocarta avait déjà été confronté à ces deux problématiques et avait fait développer par sa filiale basée à Tunis un web-SIG: GCServer ${ }^{3}$. Dans le cadre de l'ANR, nous étions confrontés aux mêmes problèmes: comment gérer les huit sites étudiés simultanément avec des acteurs de projet localisés en différents endroits en France ? Comment disposer (sans faire de demande mail) des dernières versions des données et des cartes au fur et à mesure de l'avancement du projet ? Le web-SIG s'est avéré un outil incontournable la fois pour gérer les demandes des acteurs et la complexité de chaque projet. Nous verrons qu'il accompagne le projet jusqu'à une restitution éventuelle au public.

Mais revenons au choix de la technologie Web-SIG : parmi les solutions de cartographie web, trois voies sont possibles : les logiciels clefs en main comme Google Mymap ou U-Map de la communauté OpenStreetMap ; les SIG en ligne comme Géo (anciennement DynMap) ou ArcGis Online ou QGISCloud par exemple; et enfin des serveurs/clients cartographiques.

Les raisons du choix d'une architecture client/serveur cartographique spécifique comme Chronocarto sont les suivantes :

- Les produits clefs en main sont très performants mais très basiques dans leurs fonctionnalités. Il est difficile, voire impossible, de les faire évoluer selon ses propres intérêts si ceux-ci ne coïncident pas avec ceux d'une grande communauté d'utilisateurs (ce qui n'est pas le cas en archéologie...). Des problèmes sur la confidentialité des données peuvent exister aussi.

- Les SIG en ligne, beaucoup plus puissants, sont payants pour la plupart et les licences souvent annuelles deviennent vite rédhibitoires pour un laboratoire et encore plus pour des membres associés. Il n'est donc pas possible de confier ses données et ses cartes à de telles solutions. Les changements de format des éditeurs posent aussi le problème de la pérennité des cartes créées avec ces logiciels ${ }^{4}$. Même une solution libre comme QGISCloud n'est pas gratuite si l'on doit gérer plusieurs bases de données et que l'on souhaite des cartes non publiques....

Les solutions client/serveur sont beaucoup plus souples et se basent sur des technologies libres de droit, gratuites et surtout soutenues par une vaste communauté, gage à la fois de trouver des documentations, forums et donc réponses aux problèmes rencontrés, et en perpétuelle évolution puisque soutenues par un grand nombre de développeurs suivant des normes assurant l'interopérabilité des produits. Le fait de séparer la représentation des données, côté client, de leur hébergement et chargement côté serveur permet une grande flexibilité.

\footnotetext{
${ }^{3}$ http://www.geocarta.net/html/Web-Sig.html
}

\footnotetext{
${ }^{4}$ Nous avons assisté à une conférence où la responsable d'un grand journal expliquait qu'ils n'étaient plus capables de relire les cartes créées il y a cinq ans par leur équipe. Cet obsolescence n'est pas tolérable et encore moins en Archéologie. 


\section{Historique de Chronocarto}

Chronocarto a donc été développé en 2006 comme une solution client/serveur riche utilisant plusieurs bibliothèques simultanément en fonction du type de rendu côté client (bibliothèques javascript OpenLayers ou Leaflet) et de la mise en forme des données côté serveur (Mapserver plus GeoServer). Cette solution a été développée pour des archéologues et nous avons toujours eu à l'esprit la facilité d'utilisation du produit, ne voulant pas le faire évoluer vers les potentialités d'un SIG de bureau malgré plusieurs demandes. Elle inclut toutes les fonctionnalités de base d'un SIG (Djindjian, 2008).

Chronocarto a donc été développé dans la suite de GCServer en rajoutant les notions de copyright tant sur la donnée au moment de son dépôt (possibilité d'ajouter un nom, logo, lien Internet) qu'au niveau de la carte elle-même. Un des objectifs est en effet que Chronocarto puisse devenir une véritable publication en ligne : la notion d'état de publication a été introduite permettant de passer d'un projet «en devenir », à un projet «en cours » puis un projet « achevé ». Pour tous ces stades le projet n'est toujours visualisable que par les utilisateurs authentifiés et liés au projet. Le projet peut alors basculer dans l'état «publiable en ligne » et devenir donc public et accessible grâce à une adresse unique (URL). Le portail Chronocarto actuel possède une surcouche permettant de présenter plusieurs projets à la fois qu'ils soient « en cours » (on a accès alors juste à un chapeau contenant un résumé, bibliographie, acteurs, etc.) ou « public » avec accès aux cartes.

Le transfert de Chronocarto fin 2017 des serveurs de l'ENS sur ceux de l'IN2P3 dans le cadre du consortium Huma-Num est un gage pour la pérennité des données - annoncée à 30 ans - ainsi que de la sécurité du système (passage de Windows server à Linux, protocoles sécurisés, mises à jour obligatoires des composants Java, Tomcat, MySQL, Mapserver, PHP, etc.).

Il est important de comprendre qu'un web-SIG, outil de création et partage de cartes, ne remplacera pas un SIG et qu'une phase de pré-traitement des données est la plupart du temps obligatoire avec un SIG. Mais la frontière n'est finalement pas si clairement délimitée : il est possible par exemple sous Chronocarto de numériser à l'écran et de créer des couches vectorielles ou bien de représenter directement un fichier de type tableur... De la même manière, à l'autre bout de la chaîne, ce n'est pas avec un web-SIG que l'on peut créer des cartes directement publiables dans des revues. Le passage par Adobe Illustrator ou Inkscape est souvent conseillé, même si une demande forte de mise en page directement des cartes dans Chronocarto semble émerger...

Un développement dans Chronocarto a permis d'implémenter la notion d'Atlas permettant de générer des cartes à la volée de type répartition suite à un ensemble de questions simples (Où ? Quand, Quoi ?) sur une base de données en ligne. Le résultat de ces interrogations peut être enrichi par des cartes déjà publiées (via des mots clefs).

L'accès à Chronocarto est libre mais lié à un mot de passe qu'il faut demander à l'administrateur. Néanmoins une zone libre et publique sans mot de passe a été créée permettant de faire ses premiers essais de cartographie (Chronocarto-Fab) ${ }^{5}$. Des modes d'emploi et tutoriels sont aussi disponibles en ligne dans 1'espace PSL Explore ${ }^{6}$. Un ensemble de projets en libre consultation existent classés en fonction de leur échelle (cf. infra).

Chronocarto a été développé à partir de briques open-source (GeoServer, GeoTools, Apache Tomcat, MySQL, PostgreSQL, ...) et est en développement continuel en fonction des demandes des utilisateurs. Toute bonne volonté est la bienvenue !

\footnotetext{
${ }^{5}$ http://www.chronocarto.eu/spip.php?article16\&lang=fr

6 https://explore.univ-psl.fr/fr/liblab/loutil-de-cartographie-chronofab 


\section{Potentialités de Chronocarto en tant que SIG collaboratif}

\section{Un ou des projets}

La trajectoire pour créer une carte se fait en 5 étapes :

Définition du Projet >> Introduction des Données >> Style à appliquer aux données >> Définition des données à utiliser pour les Cartes $>>$ Visualisation des Cartes.

Ceci correspond aux quatre onglets disponibles :

Projet >> Données >> Cartes >> Visualisation.

Nous détaillons ci-après la partie « Projet » :

Le web-SIG Chronocarto permet à un ensemble d'utilisateurs de publier des cartes sur la Toile. Chaque utilisateur dispose d'un espace sécurisé et peut avoir accès à un seul ou un ensemble de projets. Chaque projet (figure 1) est défini par son intitulé, le système de projection (dans la version actuelle 7.0 il ne peut pas être modifié après la création du projet), le nom du porteur de projet, son adresse électronique ainsi que le résumé-description. L'état du projet est défini à ce niveau (généralement 'En projet' ou 'En cours'). Des paramètres optionnels définis aussi par l'administrateur peuvent être sélectionnés à ce niveau pour le projet en question : possibilité d'utiliser des couches WMS, les fonds IGN, Bing, la Gestion électronique de documents, etc. Pour un projet donné, il est possible d'établir une liste de partenaires avec leur logos, leur site Internet ainsi que le type (financier/scientifique). De la même manière les 'Acteurs' d'un projet peuvent être définis. Un fil d'actualité pour chaque projet est prévu mais pas encore testé dans la version 7.0.

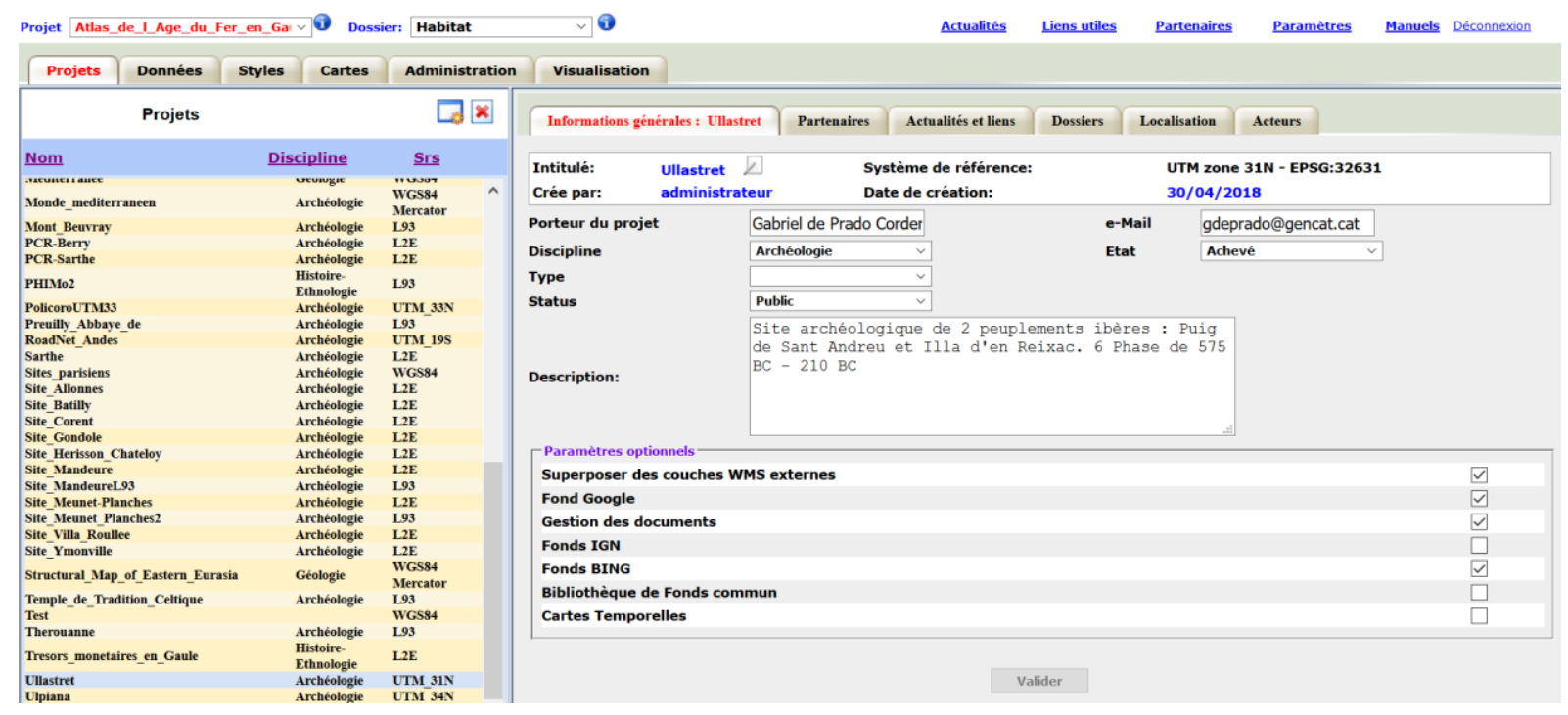

Figure 1. Définition des paramètres d'un projet (phase création)

Ces possibilités se rajoutent à celles définies par l'administrateur au moment de la création du compte d'un utilisateur (comme la possibilité d'éditer les couches, de mettre à jour des tables, des métadonnées, d'ajouter de texte à une carte, de donner accès ou non aux données, aux paramètres projets etc...). Il est donc possible de configurer complètement les droits d'un utilisateur ainsi que ceux associés à un projet donné. 


\section{Données}

Pour créer des cartes, il faut a priori des données ${ }^{7}$. Celles-ci sont téléchargées dans l'onglet données. Contrairement à un SIG, il est possible de charger des données sans référence spatiale qui pourront être associées par la suite à un endroit de la carte. Les données spatiales peuvent être vectorielles (format : shp, kml, kmz, mif) ou associées à un simple tableur (xls ou csv) dont les deux premières colonnes sont obligatoirement les coordonnées. Les données raster sont soit des fichiers avec leur fichier de géoréférencement (world), soit un geotiff ou un ArcGrid (la reprojection n'est pas implémentée dans la version actuelle et toutes les données doivent être obligatoirement dans le système de projection du projet). Pour les images géoréférencées, il est possible d'associer en métadonnée une image qui apparaitra en légende.

Lors du dépôt, une description du fichier, un logo et une adresse URL peuvent être renseignées (copyright par exemple). Les données sont associées de manière unique à un projet et même à un dossier du projet. Si des données doivent être partagées entre deux projets ou entre deux dossiers d'un même projet, il faut les dupliquer.

Les fichiers déposés (figure 2), il est possible de vérifier toutes les métadonnées et de les modifier éventuellement. Un export à ce niveau a été prévu permettant d'uploader toutes les données (si les droits ont été accordés). Lors de cette opération, les données sont compressées, peuvent être changées de système de coordonnées (pour les données vectorielles), et téléchargées, soit localement, soit envoyées par mail.

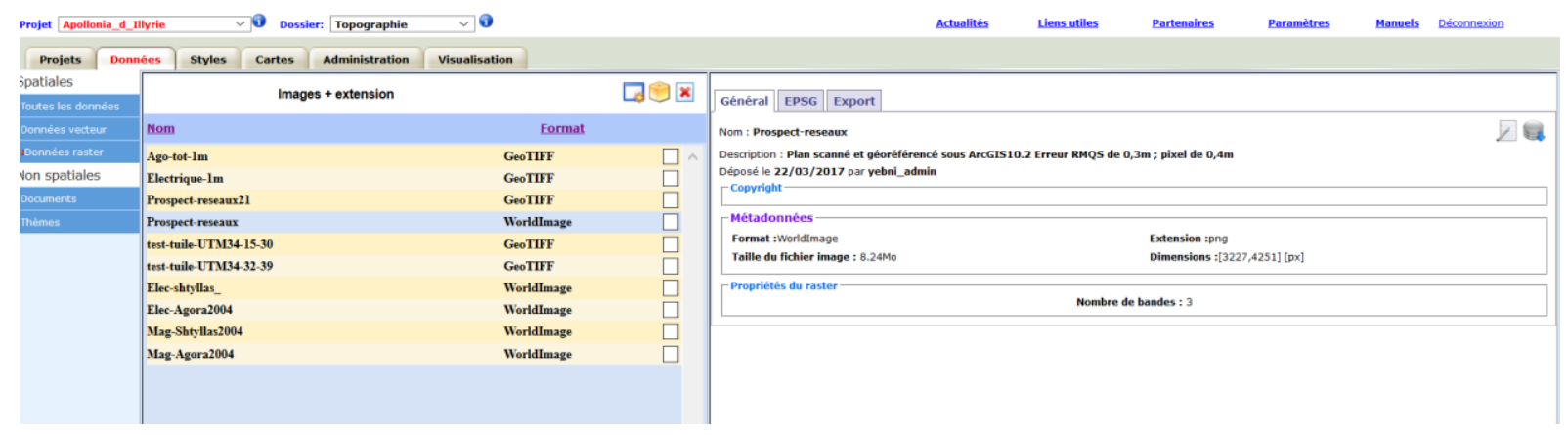

Figure 2. Création et modification des données d'un dossier d'un projet

\section{Cartes}

Dans l'onglet 'Cartes' (figure 3), il est possible de modifier, effacer ou créer une carte. Il suffit d'associer chaque donnée que l'on souhaite représenter à un style spécifique. Une fenêtre de prévisualisation permet de détecter si l'ajout de la couche est correct ou non. L'ajout d'un fond Google, Bing, IGN est possible et très utile pour vérifier les géoréférencement des couches. Il est possible de déposer un copyright pour chaque carte ainsi qu'une description.

\footnotetext{
${ }^{7}$ Bien qu'un mécanisme dans Chronocarto ('Créer un fond de carte') permettre de publier une carte juste avec un nom de localité par exemple. Une autre possibilité est d'utiliser une bibliothèque de 'Données communes' qui sont partagées par tous les projets (les fonds hydrologiques européens, les traits de côte, etc...) 


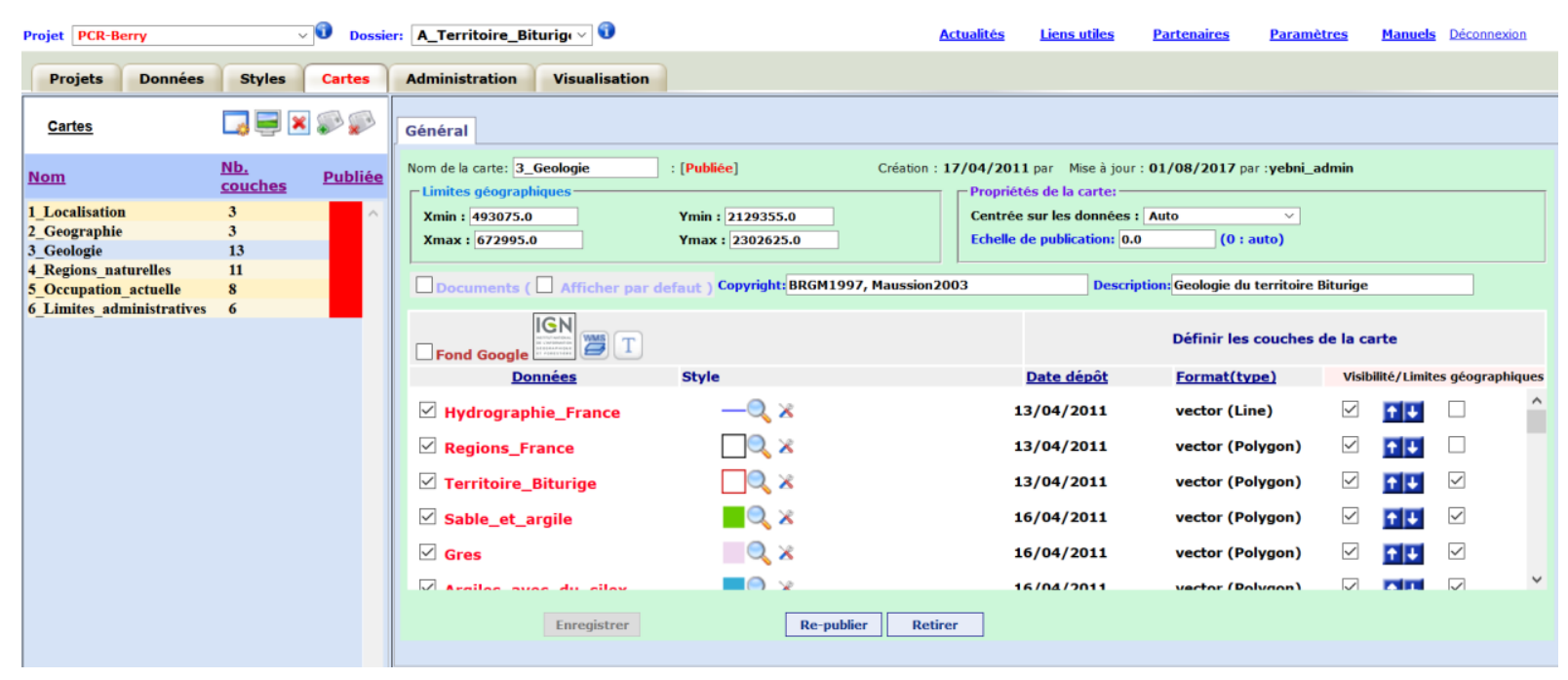

Figure 3. Création d'un ensemble de cartes dans un dossier d'un projet

\section{Visualisation}

L'onglet 'visualisation' permet de visualiser chacune des cartes d'un des dossiers d'un projet. En mode 'public', l'utilisateur n'a accès qu'à cet onglet. La première carte du dossier est automatiquement chargée. Pour les autres cartes, il suffit de cliquer dessus.

Il existe donc quand on visualise une carte, la possibilité de connaître le descriptif du projet, le descriptif du dossier courant, le résumé et le copyright de la carte en cours de visualisation ainsi que les métadonnées de chacune des couches des cartes.

Dans une carte publiée, il est possible d'accéder aux styles de chaque couche (logo 'ampoule', puis 'symbologie') et de les changer. Il est alors possible de choisir une symbologie simple : 'symbole unique' ou plus avancée: 'valeur unique', 'couleurs graduées', 'symboles proportionnels' si l'on souhaite avoir une taille ou une couleur en fonction de l'un des attributs de la couche vectorielle.

Des outils de dessin sur les cartes ont été rajoutés à droite de la carte permettant de tracer des objets géométriques simples (que l'on peut stocker comme une couche vectorielle par la suite) ainsi que du texte. Cette partie a été développée pour donner la possibilité d'un travail collaboratif sur une carte : dans le projet Celtecophys, cela nous a permis par exemple d'interpréter des cartes géophysiques et des cartes aériennes à plusieurs et à distance. On pourrait imaginer des solutions encore plus interactives avec partage de la voix et de la souris. 


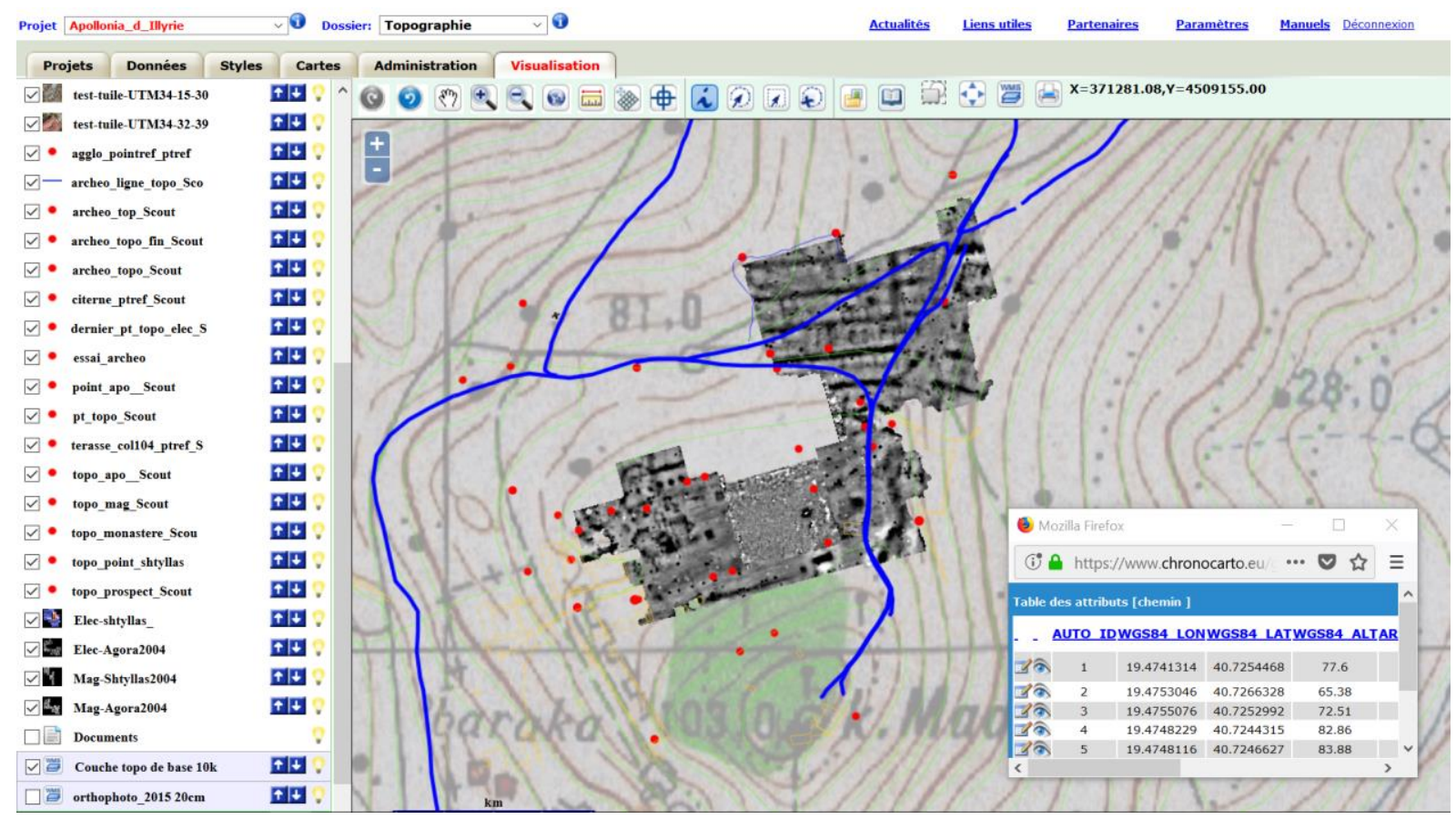

Figure 4. Visualisation d'une carte d'un dossier (ici : Topographie) d'un projet (site D'Apollonia d'lllyrie en Albanie) ainsi que de la table attributaire d'une des couches

Cette carte de travail (figure 4) générée en 2018 à partir de données obtenues sur le terrain en 2004 sur le site d'Apollonia d'Illyrie en Albanie montre le potentiel de la plateforme Chronocarto pour travailler, nous serions tenté de dire «comme sur» un SIG : placement des points de contrôle topographiques (points rouges), génération d'une trajectoire GPS (en bleu) pour vérifier le calage topographique des autres couches, placement de la carte géophysique (en densité de gris) et intégration $\mathrm{du}$ fond de carte albanais en ligne (flux WMS vers le géoportail albanais). Des requêtes spatiales peuvent être opérées sur cette carte ainsi que des requêtes et modifications sur les tables attributaires des couches (fenêtre indépendante sur la figure 4). Les résultats peuvent être exportés sous forme de tableur si les autorisations existent.

\section{Potentialités de Chronocarto pour diffuser des cartes publiques}

Une fois les cartes finalisées, voire validées par un comité éditorial, il est possible de les rendre visibles pour tout public (c'est-à-dire sans avoir à se connecter avec un mot de passe ${ }^{8}$ ). Notre laboratoire ainsi que des partenaires ont déposé depuis 2006 un ensemble de projets sur des sites archéologiques. Certains projets n'ont que quelques cartes, alors que d'autres possèdent plusieurs dossiers et plus de cinquante cartes (comme des résultats d'ANR par exemple).

Afin de mieux les présenter au public, un 'chapeau' a été créée sur la plateforme Chronocarto permettant d'établir rapidement (sous SPIP) une fiche signalétique qui renvoie à une adresse unique pour le projet que l'on souhaite rendre public (figure 5). Ces fiches sont pour l'instant regroupées en fonction de l'échelle de l'étude, des petites échelles (continent) aux grandes échelles (site). Elles peuvent être en plusieurs langues. Chaque projet ayant une URL, il est possible de l'appeler à partir de son propre site internet.

\footnotetext{
${ }^{8}$ Par rapport au paragraphe Chronocarto en tant que SIG, cela revient à n'utiliser que l'onglet « Visualisation » 


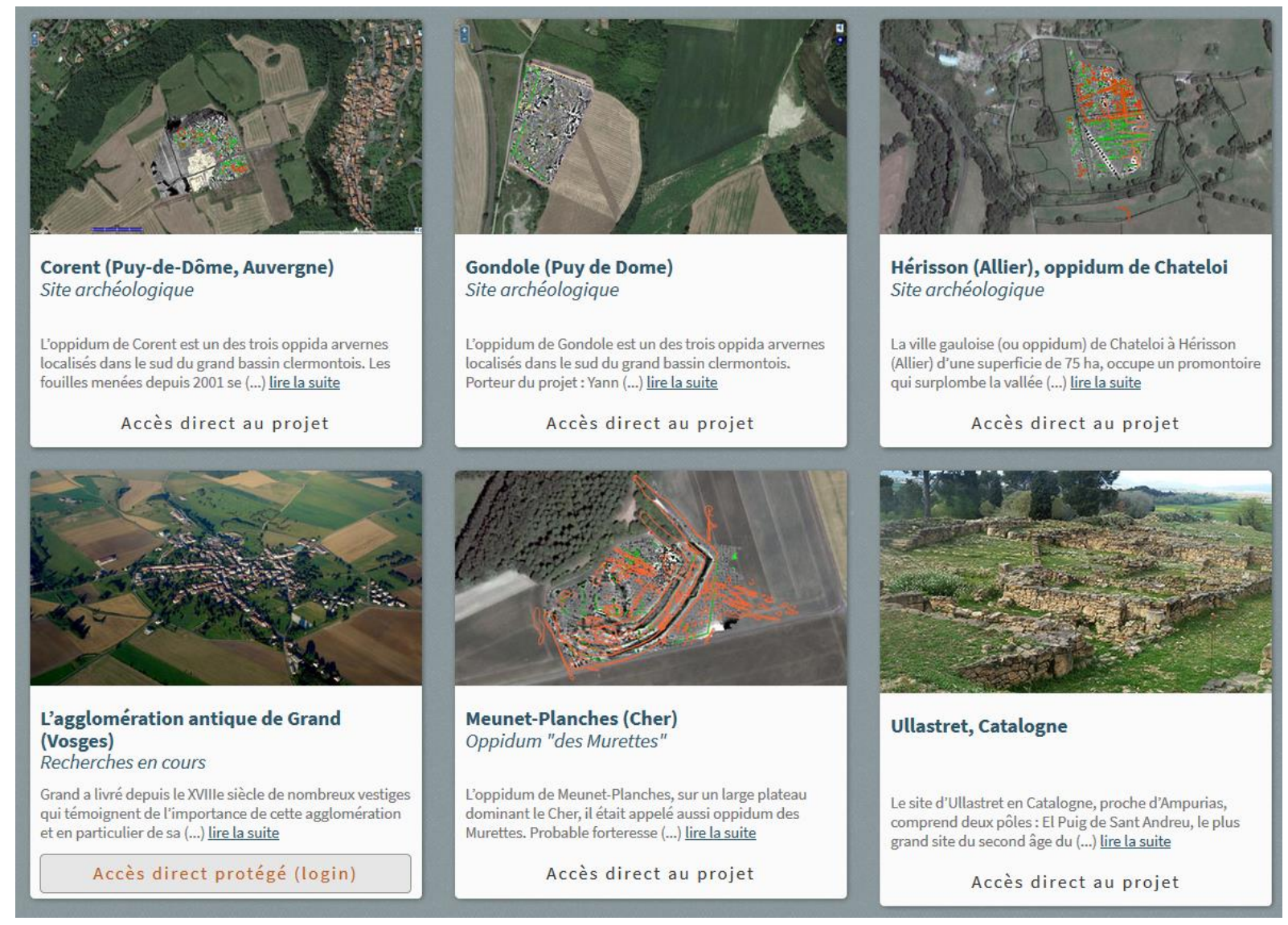

Figure 5. Interface publique de consultation des différents projets dans la plateforme Chronocarto.eu

\section{Potentialités de Chronocarto en tant qu'Atlas}

Au-delà des aspects SIG et diffusion publique, Chronocarto a aussi été développé pour créer des Atlas interactifs. Chronocarto a été conçu pour interroger en particulier la Base Fer (Batardy et al., 2008) développée dans notre laboratoire (18 000 descriptions de site). Une interface permet de poser trois niveaux de questions simples : Où (pays, région, département), Quand ? (du Bronze final jusqu'à $27 \mathrm{BC}$ ) et Quoi ? (thème : contexte ou mobilier, et éventuellement un sous-thème). Le résultat est une carte de répartition des sites (sous forme de points). La fiche synthétique de chaque site peut alors être extraite avec les références bibliographiques (figure 6). En fonction de la question, un ensemble de cartes publiées sous Chronocarto peut être proposé au lecteur (par association de mots clefs). En effet sous Chronocarto, il est possible de spécifier que certaines cartes d'un projet peuvent servir dans un Atlas et dans ce cas les associer à plusieurs mots clefs de la Base Fer (notion de carte thématique).
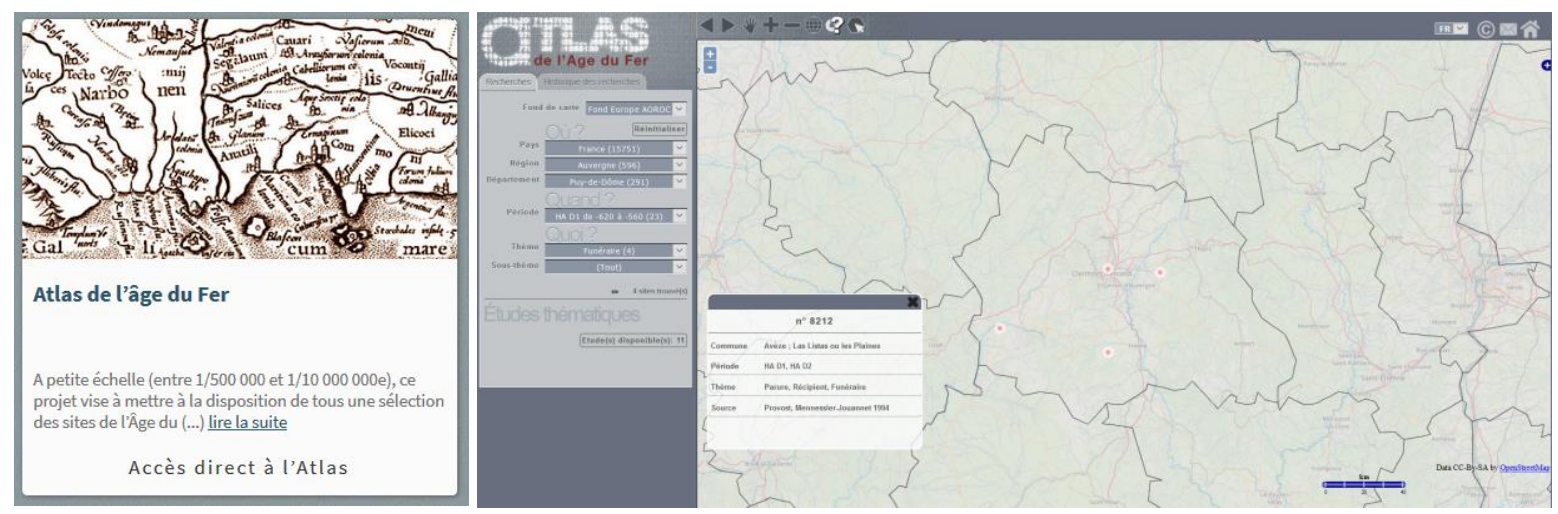

Figure 6. Atlas de l'Âge du fer en ligne. Carte de répartition des sites répondant à la question :

Sites funéraires, dans le Puy de Dôme, d'âge HA D1 ? La fiche associée à un des quatre points a été demandé. De plus onze études complémentaires (cartes thématiques) sont suggérées par rapport à cette question 
Les sites dépouillés correspondent essentiellement à la France mais les dépouillements s'étendent maintenant à toute l'Europe (Italie et Espagne en cours) en fonction des bonnes volontés... La base de données sous FileMaker et a été transférée ${ }^{9}$ sous MySQL (Cartereau, Buchsenschutz, Gruel \& Broussard, 2013) et Chronocarto travaille sur une copie de cette base. En effet, Base Fer étant alimentée au fil de l'eau, un comité valide régulièrement les modifications apportées avant que cellesci ne soient transférées dans Chronocarto.

L'interface de l'Atlas a été traduite en cinq langues ainsi que l'ontologie liée à cette base (ce qui pose les problèmes classiques de correspondance entre langues). L'ambition est de permettre à tout le monde d'utiliser cette base et de faciliter les travaux sur cette période.

D'autres Atlas correspondant à d'autres bases de données peuvent être facilement introduits.

\section{Conclusion}

Notre objectif était de créer une interface simple pour pouvoir publier aisément sur le Web des cartes issues d'études en archéologie, que ce soit une simple carte de répartition issue d'un mémoire étudiant ou le résultat d'un programme de recherche pluri-annuel comme un PCR. Chronocarto depuis sa création en 2006 a rempli cette mission et les résultats de plusieurs ANR et PCR ont été mis en ligne. Toutes les briques logicielles sont en open-source et le programme évolue en fonction de la demande des archéologues. Le champ d'application a été étendu aux Sciences de la Terre et aux Historiens. Le serveur est maintenant hébergé dans la très grande-infrastructure de recherche d'HumaNum. Intégré dans la plateforme chronocarto.eu, ce web-SIG se veut une publication en ligne qui peut être moissonnée par les moteurs de recherche.

Une évolution de Chronocarto a consisté à pouvoir lancer des requêtes simples sur une base de données et montrer une carte de répartition d'objets à la volée. Nous l'avons appliqué à la base de données de la période de l'âge du fer «BaseFer». Un atlas interactif en ligne «Atlas de l'âge du fer»a ainsi été conçu et d'autres bases de données seront interfacées prochainement. L'originalité a été d'associer à ces requêtes dynamiques des cartes thématiques déjà publiées (grâce à des mots clefs) et suggérant ainsi un niveau de lecture supplémentaire. Ce niveau correspond à une véritable publication d'une carte thématique interactive avec résumé, copyright, date et liens vers un site Web ou vers tout autre document pouvant être associé.

A l'usage, nous nous sommes rendu compte que les potentialités de Chronocarto font qu'il commence à être utilisé non plus pour publier des cartes en ligne, mais comme un simple SIG de bureau en ligne. Un des défis futurs sera de ne pas trop le compliquer pour garder sa convivialité actuelle. De nombreuses fonctionnalités restent à développer pour s'adapter aux demandes actuelles : intégration de très gros fichiers comme ceux issus du LiDAR, cartes dynamiques, interactivité avec des outils collaboratifs de pointage et liens audio-vidéo-tchat, passage au format de smartphones, lien avec un GPS, etc.

\section{Eléments de bibliographie :}

BATARDY, C., BUCHSENSCHUTZ, O., GRUEL, K., LEVERY, M. 2008. "Analyse spatiale et statistique de l'âge du Fer en France. L'exemple de la BaseFer", Revue archéologique du Centre de la France, 47. Available from: $<$ http://racf.revues.org/1159> [16 july 2014]

CARTEREAU, M., BUCHSENSCHUTZ, O., GRUEL, K., BROUSSARD, C., 2013. "Les gaulois à portée de tous, un SIG thématique sur l'âge du Fer", Conférence SIG Esri.

DJINDJIAN, F. 2008, "Webmapping in the historical and archaeological sciences. An introduction", Archeologia e Calcolatori, 19: 9-16. Available from: <http://soi.cnr.it/archcalc/indice/PDF19/1_Djindjian.pdf> [16 july 2014]

\footnotetext{
${ }^{9}$ Migration réalisée avec la participation d'Agnès Tricoche (labex TransferS CNRS ENS). 
DABAS, M. 2016. "Preventive Archaeology in France and the contribution of extensive geophysics: from ARP@ to webGIS", in : F. Boschi (éds), Looking for the future, Caring for the past, Preventive Archaeology in Theory and Practice. Bologne, Bononia University Press: 59-69. 